\title{
The comparative effectiveness of granules or blocks of superporous hydroxyapatite for the treatment of intrabony periodontal defects
}

\author{
Yoshihide Shirai $^{1,2}$, Kazuhiro Okuda ${ }^{1}$, Takehiko Kubota ${ }^{1}$, Larry F. Wolff ${ }^{3}$, Hiromasa Yoshie ${ }^{1}$ \\ ${ }^{1}$ Division of Periodontology, Department of Oral Biological Science, Graduate School of Medical and Dental Sciences, Niigata Uni- \\ versity, Niigata, Japan \\ ${ }^{2}$ Shirai Dental Clinic, Private Practice, Osaka, Japan \\ ${ }^{3}$ Division of Periodontology, Department of Developmental and Surgical Sciences, School of Dentistry, University of Minnesota, \\ Minnesota, USA \\ Email: okuda@dent.niigata-u.ac.jp
}

Received 9 February 2012; revised 14 March 2012; accepted 19 March 2012

\begin{abstract}
The aim of the present controlled clinical study was to compare the clinical response of grafting superporous hydroxyapatite (HAp) granules to superporous HAp blocks in the treatment of human intrabony periodontal defects. Twenty interproximal intrabony osseous defects in 20 healthy, non-smoking subjects diagnosed with chronic periodontitis were included in this study. These twenty subjects were randomly assigned to either the HAp granule or the HAp block groups. Clinical and radiographic measurements were determined at baseline, 3, 6, 9 and 12-month postsurgical evaluation time periods. When compared to baseline, the 12-month results indicated both treatment procedures resulted in statistically significant favorable changes in probing depth (mean value: 3.5 mm versus $3.5 \mathrm{~mm}$ ), clinical attachment level gain (3.2 $\mathrm{mm}$ versus $2.3 \mathrm{~mm}$ ) and radiographic infrabony defect depth decrease $(2.9 \mathrm{~mm}$ versus $2.5 \mathrm{~mm})$ for HAp granule and HAp block grafting respectively. At 3- and 6-months, the granule group, when compared to the block group, exhibited a statistically significantly more favorable clinical response in clinical attachment level $(4.1 \mathrm{~mm}$ versus $5.9 \mathrm{~mm}, \mathrm{p}<0.05$ at 3 -months; $4.3 \mathrm{~mm}$ versus $6.5 \mathrm{~mm}, \mathrm{p}<0.01$ at 6 months). The present study demonstrated that both grafting of superporous HAp granules and grafting of HAp blocks were similarly successful in the treatment of human intrabony periodontal defects.
\end{abstract}

Keywords: Superporous Hydroxyapatite; Granule; Block; Randomized Controlled Clinical Study

\section{INTRODUCTION}

To regenerate periodontal osseous defects caused by chronic periodontitis is a very important outcome of periodontal therapy. A variety of graft materials have been widely embraced for being used in periodontal regenerative therapy. Autogenous bone, allogeneic bone, and artificial bone have been used to reconstruct lost periodontal defects; however, the use of autogenous bone involves high surgical invasiveness because of the need to often harvest the bone from a separate surgical site. The use of allogeneic bone is sometimes perceived in being associated with infections, raises ethical concerns, and for these reasons may not always be widely used, such as in Japan. Artificial bone has several advantages, including no need for bone harvesting from a separate surgical site or cadaver, excellent biocompatibility, and a relatively user friendly surgical procedure. Design requirements for artificial bone include surgical manipulability, structural compatibility with the area exhibiting the osseous defect, support properties of the graft material, and the ability to induce bone regeneration. However, no artificial bone meeting all these clinical/structural requirements has yet been developed. Artificial bone is used in many patients in our clinic, and we have been active in developing the next generation of artificial bone with more favorable clinical properties.

Hydroxyapatite (HAp) has high biocompatibility and good bioaffinity, stimulates osteoconduction, and is slowly replaced by the host bone after implantation [1-8]. Since early in the 1980s, blocks and granules of porous HAp have been widely used as a bone substitute in the fields of orthopedic, craniofacial, and periodontal surgical therapy. However, in order to establish an ideal bone substitute to induce new bone, it is necessary that it has a structure with the ability of osteogenic cells being able to easily penetrate deeply into the HAp osseous grafting materials. Recently, superporous HAp has been developed, which has a high level of porosity of $85 \%$ and a 
more ideal interconnection among the macropores within it (APACERAM-AX ${ }^{\circledR}$, HOYA Co., Tokyo, Japan). Several in vitro studies [9-12] reported that osteogenic cell penetration could be significantly improved. Therefore, it is expected this kind of superporous HAp would serve as a more optimally functional scaffold for preparation of tissue-engineered bone and contribute to a greater quality of bone repair/regeneration.

When superporous HAp is applied clinically, it is important to know which type of HAp osseous graft material, granules or blocks, is more suitable as the best treatment for achieving the most favorable clinical results. Granules are easy to place into periodontal intrabony defects, however, the HAp granules may have a tendency to migrate from the defect until at least the initial stages of wound healing have been completed. On the other hand, HAp osseous blocks may be difficult to adapt to the morphology of the defect, although osseous HAp blocks are usually easily maintained in the involved site during the healing process.

Therefore, the aim of this randomized controlled clinical study was to compare the clinical efficacy of grafting with superporous HAp granules or HAp blocks in the treatment of human intrabony periodontal defects.

\section{MATERIALS AND METHODS}

\subsection{Study Populations}

Twenty patients ( 18 females and 2 males, mean age 61.1 \pm 6.2 years) with moderate to advanced chronic periodontitis, who were scheduled to receive periodontal therapy at Shirai Dental Clinic, were recruited between September 2008 and November 2011 for this comparative controlled clinical study. Twenty patients who met the inclusion criteria for entry into the surgical phase of this study were divided into one of the two study groups, the granule or block study groups, taking into consideration the patient's age, the number of osseous walls and the specific teeth needing treatment.

Prior to initiating this study, the patients were informed of the purpose and design of this clinical trial and were required to sign an informed consent. The format of the study was reviewed and approved by the Research Ethics Committee of Niigata University Faculty of Dentistry (No.20-R11-08-04-m, on August 11, 2008) in accordance with the Helsinki Declaration of 1975 and as revised in 2008.

The criteria for inclusion of patients and periodontal sites in this study were individuals who 1) were nonsmoking, free of systemic complications and had no history of allergies; 2) did not use antibiotics over the previous 6 months prior to treatment; 3) had no treatment for periodontitis during the previous 2 years; 4 ) had one infrabony defect with a probing depth $(\mathrm{PD}) \geq 4 \mathrm{~mm}$, clinical attachment level (CAL) $\geq 4 \mathrm{~mm}$, an osseous defect depth estimated to be $\geq 3 \mathrm{~mm}$ when measured radiographically and 5) had at least $2 \mathrm{~mm}$ of keratinized gingiva on the facial aspect of the selected tooth.

\subsection{Presurgical Therapy}

Prior to the surgical procedures, initial periodontal therapy consisted of stringent plaque control repeated until patients achieved a Modified O'Leary plaque score [13] of $\leq 10 \%$, full-mouth scaling and root planing under local anesthesia and occlusal adjustment if trauma from occlusion was present. After completion of the initial therapy, a reevaluation periodontal examination was performed 3 months later to determine patient response to the therapy and to confirm the need for periodontal surgery. Patients who met all criteria for entry into the surgical phase of the study were then divided into two groups, the granule or block study groups considering patients age, number of osseous walls, and treated teeth.

\subsection{Clinical Parameters}

A clinical examination was performed by a single examiner (author YS) at baseline and at 3, 6, 9 and 12 months after the surgical procedure. Patient oral hygiene status was evaluated by the plaque index (PI) [14], as an expression of the level of an individual's supragingival plaque accumulation. Gingival inflammation was assessed by the gingival index (GI) [15] and bleeding on probing (BOP) was recorded as absent (0) or present (1). In addition, the following clinical parameters were evaluated to assess the healing results in the two study groups. Probing depth (PD) was measured from the gingival margin to the base of the pocket and clinical attachment level (CAL) was measured from the cementalenamel junction to the base of the pocket. All clinical measurements (PD, CAL) were made using a calibrated color-coded periodontal probe (PCPUNC15, Hu-Friedy Mfg. Co., Inc., Chicago, IL) to the nearest $\mathrm{mm}$ and customized acrylic stents with a guiding groove. The calibration was performed just prior to the start of the study with 5 volunteer subjects at Niigata University Faculty of Dentistry. The reproducibility of the clinical measurements was calculated, and an agreement or reproducibility value of 0.857 was obtained for CAL with a difference of $\pm 1 \mathrm{~mm}$. This provided well-defined and highly reproducible clinical measurements at each granule- and block-treated site and for each examination time point, baseline and 3, 6, 9 and 12 months. The 12-month clinical results at each treated intrabony osseous defect site were assessed and the difference between baseline and 12 months for the clinical values (PD, CAL) was determined. 


\subsection{Radiographic Examination}

A commercially available film holder device (CID-2, Hanshin Technical Laboratory, Ltd., Hyogo, Japan) was modified by placing registration material (UNIFAST II ${ }^{\circledR}$, GC Co., Tokyo, Japan) on the bite blocks to index the dentition. Standardized reproducible radiographs using a paralleling cone technique with positioning aids were taken at each treated granule and block site at baseline and 3, 6, 9 and 12 months after surgery. All radiographs were evaluated by a single examiner (author $\mathrm{KO}$ ) who was masked to the treatment group for which a patient was assigned. Radiographic infrabony defect depth (IBD) was assessed using the method described by Cardaropoli and Leonhardt [16]. Briefly, the IBD was measured as the radiographic difference in vertical dimension between the projection of the osseous crest adjacent to the root surface (BCP) and the most coronal osseous level adjacent to the root surface where the periodontal ligament space was considered to have a normal width $(\mathrm{BoBD})$ or IBD $=\mathrm{BCP}-\mathrm{BoBD}$.

\subsection{Treatment Procedures}

Periodontal surgical procedures were performed on an outpatient basis under aseptic conditions by one trained periodontal clinician (author YS). After providing local anesthesia to patients, crevicular incisions were made and full-thickness mucoperiosteal flaps were elevated. Vertical releasing incisions were performed only if necessary for better access or to achieve more favorable closure of the surgical site. The surgical procedure fully exposed the intrabony defects and preserved the marginal gingiva and interdental tissue. Meticulous defect debridement and root planing were carried out to remove visible subgingival plaque, calculus, inflammatory granulation tissue and pocket epithelium. The surgical sites were thoroughly rinsed with sterile saline and care was taken to keep the area free of saliva and blood.

In the granule-treated sites, the HAp granules were then placed using amalgam condensers into the defects to the vertical height of the corresponding adjacent bone level of the intrabony defect. In the block-treated sites, the HAp block was trimmed to meet the morphological size of the osseous defect site as precisely as possible, and then the block graft was inserted into the osseous defect to the vertical height of the corresponding adjacent bone level of the intrabony defect. The surgical flaps were repositioned to their presurgical levels and sutured in the majority of the cases with the 5-0 nylon suture (Softretch $^{\circledR}$, GC Co., Tokyo, Japan) and the remainder were sutured with 4-0 silk suture (NESCO SUTURE ${ }^{\mathbb{B}}$, AZWELL Inc., Osaka, Japan) utilizing an interrupted, vertical mattress technique. Representative clinical cases of grafting with HAp granules or HAp blocks surgically treated sites are presented in Figures 1 and 2. Postoperative care included systemic administration of cefaclor at $750 \mathrm{mg}$ per day for five days and $0.12 \%$ fradiomycin sulfate rinses (DENTARGLE ${ }^{\circledR}$ gargle, SHOWA YAKUHIN KAKO Co., Ltd., Tokyo, Japan) three times daily for six weeks. Sutures were removed at 10 days postsurgery. After suture removal, patient plaque control using the roll tooth brushing technique with an ultra soft toothbrush (Butler gum ${ }^{\circledR}$ classic 333 ultra-soft 3-row, John. O. Butler, Co., Chicago, IL) was resumed at the surgically treated sites. Supragingival professional tooth cleaning was also performed weekly for the first six weeks postsurgery and thereafter the patients were recalled once a month for up to 12 months post-surgery for oral hygiene reinforcement and prophylaxis.

\subsection{Statistical Analysis}

Results were averaged (mean \pm standard deviation) for the clinical and radiographic parameters. These data were statistically evaluated by a non-parametric test using a commercially available software program (Stat-view ${ }^{\circledR}$ version J-5.0, SAS Institute Inc., Cary, NC). Taking into
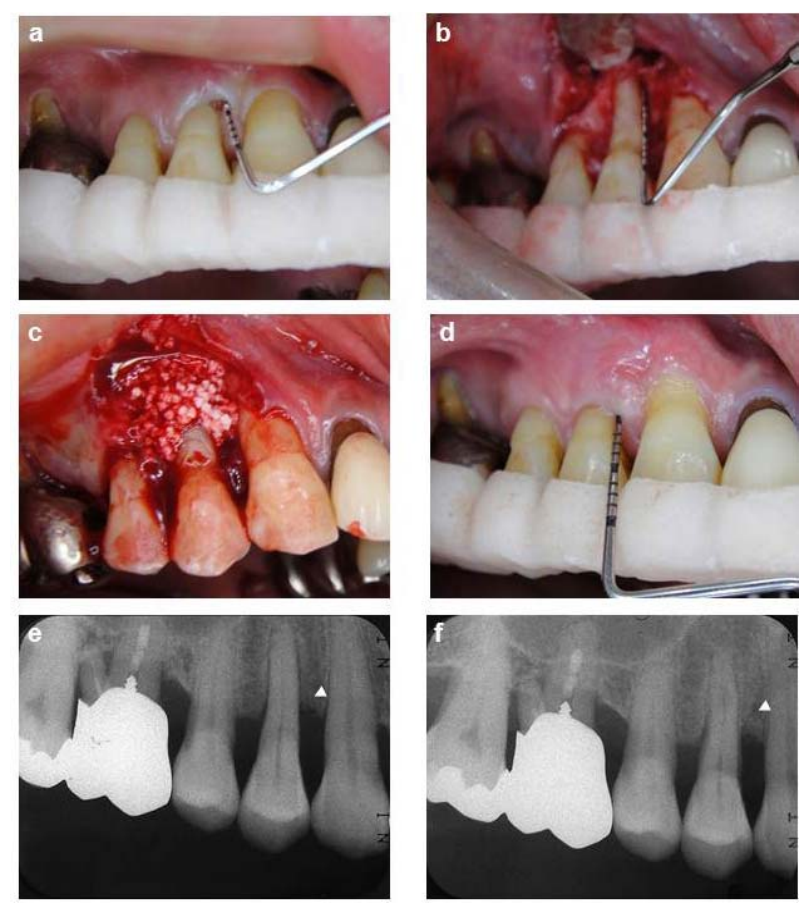

Figure 1. Representative clinical case of a site surgically treated with HAp-granules. A deep probing depth was detected on the mesial surface of maxillary left first premolar (a). The defect demonstrated a 2-wall component (b), Grafting with a HAp-granule into the intrabony osseous defect (c), Clinical illustration 12 months after surgical treatment (d), Pretreatment standardized radiograph suggests an intrabony defect (arrowhead) on the mesial aspect (e), Twelve-month post-treatment standardized radiograph depicts defect fill (arrow-head) on the mesial aspect consistent with the clinical findings (f). 

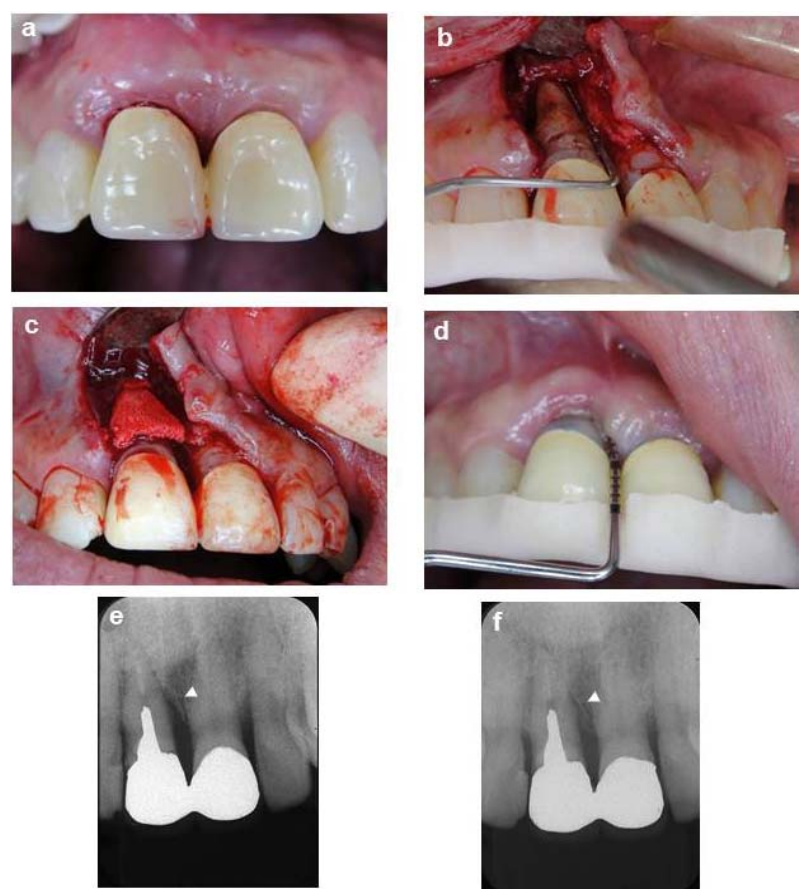

Figure 2. Representative clinical case of a site surgically treated with HAp-blocks. A deep probing depth was detected on the mesial site of the maxillary right central incisor (a). The defect demonstrated a 2-wall component (b), Grafting with a Hap-block into the intrabony osseous defect (c), Clinical illustration 12 months after surgical treatment (d), Pretreatment standardized radiograph suggests an intrabony defect (arrowhead) on the mesial aspect (e), Twelve-month post-treatment standardized radiograph depicts defect fill (arrow-head) on the mesial aspect consistent with the clinical findings (f).

account the paired nature of the changes from baseline to 12 months in each group, the Wilcoxon signed-rank matched pair test was performed for the pairwise statistical analysis of these data. The Mann-Whitney U-test was applied to compare clinical and radiographic outcomes between the granule and the block groups at baseline and 3, 6, 9 and 12 months. The null hypothesis was rejected when the risk percentage was below $5 \%(\mathrm{p}$ $<0.05)$.

\section{RESULTS}

The 20 patients ( 2 males/18 females) enrolled in this clinical trial returned for all scheduled maintenance visits. Throughout the study period, there were no infectious episodes and no other adverse complications in either the granule or block treated sites. The soft tissue healing response at all treated sites was excellent and patients were very comfortable during and following treatment.

\subsection{Study Population and Defect Characteristics}

The distribution of all patients with respect to age and gender, number of osseous walls in the treated defects, and number of treated teeth are shown in Tables $\mathbf{1}$ and 2.

\subsection{Oral Hygiene Level and Infection Control}

At baseline and at 12 months, the mean PI, GI and BOP demonstrated no statistically significant differences between the granule and block groups $(p>0.05)$. At 12 months following therapy, in the granule and the block study groups, the mean PI and GI were zero for both study groups, whereas the BOP was $0.3 \pm 0.5$ in the block group. These values were not statistically significantly different when compared to baseline $(p>0.05)$.

\subsection{Clinical and Radiographic Changes at $\mathbf{1 2}$ Months}

\subsubsection{Cross-Sectional Comparisons between Study Groups}

The mean clinical and radiographical changes at baseline and 3, 6, 9 and 12 months for the granule and the block study groups are shown in Table 3 . Between group comparisons at baseline for the PD, CAL, and IBD demonstrated no statistically significant differences $(\mathrm{p}>$ 0.05 ) between the granule and the block study groups. At 3 and 6 months, only the CAL in the granule study group exhibited statistical significance when compared to the block study group ( 3 months: $4.1 \pm 1.4$ vs $5.9 \pm 1.7$, p <

Table 1. Patient age, gender, number of osseous walls, and treated teeth for the granule and block study groups.

\begin{tabular}{ccc}
\hline Characteristic & Granule & Block \\
\cline { 2 - 3 } Male & $(\mathrm{n}=10)$ & $(\mathrm{n}=10)$ \\
\hline Female & $61.6 \pm 7.4$ & $69.6 \pm 5.1$ \\
Osseous Walls & 2 & 0 \\
1-walled defect & 8 & 10 \\
2-walled defect & & \\
3-walled defect & 0 & 0 \\
Treated Teeth & 4 & 6 \\
Maxillary incisors & 6 & 4 \\
Maxillary premolars & & 5 \\
Maxillary molars & 5 & 3 \\
Mandibular incisors & 1 & 0 \\
Mandibular premolars & 0 & 0 \\
Mandibular molars & 2 & 2 \\
\hline
\end{tabular}


Table 2. Distribution of number of osseous walls and treated teeth for the granule and block study groups.

\begin{tabular}{ccccc}
\hline \multirow{2}{*}{ Patients } & \multicolumn{2}{c}{ Granule } & & \multicolumn{2}{c}{ Block } \\
\cline { 2 - 5 } & Teeth & Osseous Walls & Teeth & Osseous Walls \\
\hline 1 & Maxillary incisor & 2 walled defect & Mandibular premolar & 2 walled defect \\
2 & Maxillary premolar & 2 walled defect & Maxillary premolar & 2 walled defect \\
3 & Maxillary incisor & 3 walled defect & Maxillary premolar & 3 walled defect \\
4 & Maxillary premolar & 3 walled defect & Maxillary incisor & 3 walled defect \\
5 & Maxillary premolar & 3 walled defect & Maxillary premolar & 3 walled defect \\
6 & Maxillary premolar & 3 walled defect & Maxillary incisor & 3 walled defect \\
7 & Mandibular premolar & 2 walled defect & Maxillary incisor & 2 walled defect \\
8 & Maxillary molar & 3 walled defect & Maxillary incisor & 3 walled defect \\
9 & Mandibular premolar & 2 walled defect & Maxillary incisor & 2 walled defect \\
\hline
\end{tabular}

Table 3. Mean $( \pm \mathrm{sd})$ clinical and radiographic measurements at baseline, 3 months, 6 months, 9 months and 12 months for the granule and block study groups $(\mathrm{n}=10$ subjects in each treatment group).

\begin{tabular}{|c|c|c|c|c|c|c|c|}
\hline \multirow{2}{*}{$\begin{array}{l}\text { Clinical Index }{ }^{*} \text { and } \\
\text { Treatment Group }\end{array}$} & \multirow{2}{*}{ Baseline (BL) } & 3 & 6 & 9 & \multirow{2}{*}{12 Months } & BL vs 12 Months & \multirow{2}{*}{$\begin{array}{c}\Delta \mathrm{BL} \text { and } 12 \\
\text { months }\end{array}$} \\
\hline & & Months & Months & Months & & p-Value ${ }^{\dagger}$ & \\
\hline \multicolumn{8}{|l|}{ PD } \\
\hline Granule & $7.4 \pm 2.8$ & $3.1 \pm 1.2$ & $3.6 \pm 0.5$ & $3.9 \pm 1.3$ & $3.9 \pm 1.0$ & $\mathrm{p}<0.01$ & $3.5 \pm 2.4$ \\
\hline Block & $7.5 \pm 2.2$ & $4.7 \pm 1.6$ & $5.0 \pm 1.7$ & $4.4 \pm 1.4$ & $4.0 \pm 0.9$ & $\mathrm{p}<0.01$ & $3.5 \pm 2.2$ \\
\hline p-Value & NS & NS & NS & NS & NS & & NS \\
\hline \multicolumn{8}{|l|}{ CAL } \\
\hline Granule & $8.1 \pm 3.1$ & $4.1 \pm 1.4$ & $4.3 \pm 0.8$ & $4.7 \pm 1.3$ & $4.9 \pm 1.1$ & $\mathrm{p}<0.05$ & $3.2 \pm 2.4$ \\
\hline Block & $8.4 \pm 2.3$ & $5.9 \pm 1.7$ & $6.5 \pm 1.7$ & $5.8 \pm 2.3$ & $5.8 \pm 1.9$ & $\mathrm{p}<0.05$ & $2.3 \pm 2.8$ \\
\hline p-Value & NS & $\mathrm{p}<0.05$ & $\mathrm{p}<0.01$ & NS & NS & & NS \\
\hline \multicolumn{8}{|l|}{ IBD } \\
\hline Granule & $4.9 \pm 1.7$ & $2.9 \pm 1.3$ & $2.5 \pm 1.4$ & $2.1 \pm 1.3$ & $2.0 \pm 1.3$ & $\mathrm{p}<0.01$ & $2.9 \pm 0.9$ \\
\hline Block & $5.0 \pm 1.4$ & $3.3 \pm 1.5$ & $2.8 \pm 1.9$ & $2.8 \pm 1.4$ & $2.5 \pm 1.3$ & $\mathrm{p}<0.01$ & $2.5 \pm 1.4$ \\
\hline p-Value & NS & NS & NS & NS & NS & & NS \\
\hline
\end{tabular}

${ }^{*} \mathrm{PD}=$ Probing Depth, CAL = Clinical Attachment Level, IBD = Radiographic Infrabony Defect Depth. ${ }^{\dagger}$ Represents statistical significance level between baseline and 12 months in each treatment group. Statistical significant, $p<0.01$ or $p<0.05$. Represents statistical significance level between study groups at baseline and, 3, 6, 9 and 12 months. NS = not significant, $\mathrm{p}>0.05$.

0.05 ; 6 months: $4.3 \pm 0.8$ vs $6.5 \pm 1.7, \mathrm{p}<0.01)$. At 12 months, the mean PD, CAL and IBD parameters demonstrated no statistically significant differences $(p>0.05)$ between the granule and the block study groups (PD: 3.9 \pm 1.0 vs $4.0 \pm 0.9$; CAL: $4.9 \pm 1.1$ vs $5.8 \pm 1.9$; IBD: 2.0 \pm 1.3 vs $2.5 \pm 1.3$ ).

\subsubsection{Longitudinal Comparisons of Each Study Group}

When baseline was compared to 12 months, the PD de- creased significantly in each study group $(\mathrm{p}<0.01)$. At 12 months, when compared to baseline, the CAL in the granule and block study groups decreased from $8.1 \pm 3.1$ $\mathrm{mm}$ to $4.9 \pm 1.1 \mathrm{~mm}$ and $8.4 \pm 2.3 \mathrm{~mm}$ to $5.8 \pm 1.9 \mathrm{~mm}$, respectively. There was a significant gain $(p<0.05)$ in CAL at 12 months when compared to baseline in both study groups. The radiographic IBD was significantly decreased at 12 months when compared to baseline, from $4.9 \pm 1.7 \mathrm{~mm}$ to $2.0 \pm 1.3 \mathrm{~mm}(\mathrm{p}<0.01)$ in the granule study group and from $5.0 \pm 1.4 \mathrm{~mm}$ to $2.5 \pm 1.3$ 
$\mathrm{mm}(\mathrm{p}<0.01)$ in the block study group.

Comparison between the granule and the block study groups for change in mean clinical and radiographic measurements from baseline to 12 months is also shown in Table 3. The change between study groups from baseline to 12 months in PD, CAL and IBD of the granule and block study groups were not statistically significantly different $(\mathrm{p}>0.05)$.

\section{DISCUSSION}

Patient age and teeth with osseous defects treated in both study groups were similar at baseline. Each subject participating in the clinical investigation demonstrated excellent oral hygiene throughout the entire study. Results from this investigation showed that both regenerative procedures, the grafting of superporous HAp granules or blocks, resulted in significant clinical improvement in the treatment of intrabony periodontal defects in terms of $\mathrm{PD}$ reduction, CAL gain, and radiographic bone fill between baseline and 12 months.

We have previously reported the clinical effectiveness of HAp granules without interconnections and exhibiting a porosity of $15 \%$ on periodontal tissue regeneration [17]. This bone grafting material was a former type of superporous HAp granules, and manufactured by the same company which developed superporous hudroxyapatite. Regarding granule type, we compared the clinical results of our present investigation to our previous results.

There was no significant difference of PD reduction between superporous type and porous type of the HAp bone grafting material during one year after treatment (superporous type: $3.5 \pm 2.4 \mathrm{~mm}$ versus porous type: 3.7 $\pm 2.0 \mathrm{~mm}$ ). However, in regards to clinical attachment gain and radiographic osseous defect fill, the superporous HAp bone graft gave clinically superior results (CAL: $3.2 \pm 1.2 \mathrm{~mm}$, IBD: $2.9 \pm 0.9 \mathrm{~mm}$ ) when compared to the porous HAp bone graft results (CAL: $2.0 \pm$ $2.4 \mathrm{~mm}$, IBD: $2.7 \pm 1.6 \mathrm{~mm}$ ).

These findings may be explained by the fact that newly developed superporous HAp has $85 \%$ porosity and interconnected pores on the inside and this bone structure will allow significant penetration of the osteoblasts into the bone graft material. It is noteworthy the osteoblasts penetrating into the porous bone maintains high alkaline phosphatase activity during the culture period [9]. In our previous study, the cell-superporous HAp complex using periosteal cells, when compared to the cell-free superporous HAp, formed osteoid in pore regions of the superporous HAp [18]. These in vitro experimental results could explain one of the likely reasons the superporous HAp favors the clinical results we see in the treatment of periodontal osseous defects with both the granule and block superporous HAp bone grafting material.
The granule treatment group, when compared to the block study group, provided a statistically significantly more favorable clinical response in terms of CAL value $(p<0.05)$ at 3 and 6 months post-surgery. The benefit of clinically using HAp granules, when compared to block HAp bone, is that the granule will easily fill and adapt to a complex periodontal osseous defect. Therefore, at 3 and 6 months in the early healing periods after surgery, osteoconductivity is induced and may lead to earlier clinical attachment gain, when compared to the HAp blocks. On the other hand, it is necessary to trim the block graft and it is more difficult to adapt the HAp block graft to the morphology of the osseous defect, however, it is easy to keep the HAp block graft material in the osseous defect until tissue healing has occurred. To gain structural compatibility with the osseous defect, it can be expected computer-aided design (CAD) and computer aided manufacture (CAM) technology be introduced in this field. Recently, in the field of the restorative dentistry, Ender and Mörmann et al. succeeded in automatically reproducing a morphology matching the natural surface left after preparation by milling and grinding a ceramic block based on digital three-dimensional (3D)-data [19]. This novel method would have promise in meeting the requirements to precisely shape an osseous block graft in being prepared for a specific morphology of a complex osseous defect.

The ideal graft material such as tissue-engineered bone would be expected to have a clinical property which would permit the penetration of osteogenic cells into pore regions of block bone grafts. In oral regions, periosteal cells are candidates for osteogenic cells sources. Yamamiya and Okuda et al. established the methodology of autologous implantation of cultured periosteal sheets, in combination with HAp granules and platelet-rich plasma for periodontal regenerative therapy. The effectiveness and safety of this therapeutic methodology were demonstrated in clinical studies [20,21].

Our research group has already succeeded in developing a methodology to maintain periosteal cells by a 3D high-density culture system using acid-treated superporous HAp blocks, in vitro. The use of human periosteum-derived cells combined with superporous HAp blocks has significant potential as an osteogenic bone substitute for periodontal regenerative therapy in humans. In the future, the osteogenic bone substitute described above could be milled and ground to a specific size and shape so that it precisely adapts to a specific osseous defect morphology using CAD/CAM technology. This in turn has the potential to lead to improved and more superior clinical results in the treatment of our patients.

\section{CONCLUSION}

Within the limits of the current study, it can be con- 
cluded that grafting using both superporous HAp granules and HAP blocks were similarly successful in the treatment of human intrabony defects.

\section{ACKNOWLEDGEMENTS}

This study was supported in part by a Grant-in Aids for Scientific Research from the Japan Society for the promotion of Science (17390558), Tokyo, Japan. Drs. Shirai, Okuda, Kubota, Wolff and Yoshie report no financial relationships related to any products involved in this study. Dr. Wolff played a role in study design along with reviewing and editing the manuscript.

\section{REFERENCES}

[1] Bucholz, R.W., Carlton, A. and Holmes, R.E. (1987) Hydroxyapatite and tricalcium phosphate bone graft substitute. Orthopedic Clinics of North America, 18, 323-334.

[2] Holmes, R.E., Bucholz, R.W. and Monney, V. (1986) Porous hydroxyapatite as a bone-graft substitute in metaphyseal defects. The Journal of Bone and Joint Surgery, American Volume, 68A, 904-911.

[3] Ishihara, K., Arai, H., Nakabayashi, N., Morita, S. and Furuya, K. (1992) Adhesive bone cement containing hydroxyapatite particle as bone compatible filler. Journal of Biomedical Materials Research, 26, 937-945. doi: $10.1002 / \mathrm{jbm} .820260708$

[4] Ohgushi, H., Goldberg, V.M. and Caplan, A.I. (1989) Repair of bone defects with marrow cells and porous ceramic, experiments in rats. Acta Orthopedica Scandinavica, 60, 334-339. doi:10.3109/17453678909149289

[5] Radin, S.R. and Ducheyne, P. (1994) Effect of bioactive ceramic composition and structure on in vitro behavior, III. Porous versus dense ceramics. Journal of Biomedical Materials Research, 28, 1303-1309. doi: $10.1002 / \mathrm{jbm} .820281108$

[6] Tsuruga, E., Takita, H., Itoh, H., Wakisaka, Y. and Kuboki, Y. (1997) Pore size of porous hydroxyapatite as the cell-substratum controls BMP-induced osteogenesis. Journal of Biochemistry, 121, 317-324. doi:10.1093/oxfordjournals.jbchem.a021589

[7] Yamamura, K., Iwata, H. and Yotsuyanagi, T. (1992) Synthesis of antibiotic-loaded hydroxyapatite beads and in vitro drug release testing. Journal of Biomedical Materials Research, 26, 1053-1064.

[8] Redey, S.A., Razzouk, S., Rey, C., Bemache-Assollant, D., Leroy, G., Nardin, M. and Coumot., G. (1999) Osteoclast adhesion and activity on synthetic hydroxyapatite, carbonated hydroxyapatite, and natural calcium carbonate: relationship to surface energies. Journal of Biomedical Materials Research, 45, 140-147. doi:10.1002/(SICI)1097-4636(199905)45:2<140::AID-J BM9>3.0.CO;2-I

[9] Sakamoto, M., Nakasu, M., Matsumoto, T. and Okihana, H. (2007) Development of superporous hydroxyapatites and their examination with a culture of primary rat osteoblasts. Journal of Biomedical Materials Research, Part A, 82, 238-242. doi:10.1002/jbm.a.31013

[10] Yoshikawa, H. and Myoui, A. (2005) Bone tissue engi- neering with porous hydroxyapatite ceramics. Journal of Artificial Organs, 8, 131-136.

doi:10.1007/s10047-005-0292-1

[11] Agata, H., Asahina, I., Yamazaki, Y., Uchida, M., Shinohara, Y., Honda, M., Kagami, H. and Ueda, M. (2007) Effective bone engineering with periosteum-derived cell. Journal of Dental Research, 86, 79-83. doi:10.1177/154405910708600113

[12] Matsushima, A., Kotobuki, N., Tadokoro, M., Kawate, K., Yajima, H., Takakura, Y. and Ohgushi, H. (2009) In vivo osteogenic capacity of human mesenchymal cells cultured on hydroxyapatite and on beta-tricalcium phosphate. Artificial Organs, 33, 474-481. doi:10.1111/j.1525-1594.2009.00749.x

[13] O’Leary, T.J., Drake, R.B. and Naylor, J.E. (1972) The plaque control record. Journal of Periodontology, 43, 38. doi:10.1902/jop.1972.43.1.38

[14] Silness, J. and Löe, H. (1964) Periodontal disease in pregnancy. II. Correlation between oral hygiene and periodontal condition. Acta Odontologica Scandinavica, 22, 121-135. 121-135. doi:10.3109/00016356408993968

[15] Löe, H. and Silness, J. (1963) Periodontal disease in pregnancy. I. Prevalence and severity. Acta Odontologica Scandinavica, 21, 533-551. doi:10.3109/00016356309011240

[16] Cardaropoli, G. and Leonhardt, A.S. (2002) Enamel matrix proteins in the treatment of deep intrabony defects. Journal of Periodontology, 73, 501-504. doi:10.1902/jop.2002.73.5.501

[17] Okuda, K., Tai, H., Tanabe, K., Suzuki, H., Sato, T., Kawase, T., Saito, Y., Wolff, L.F. and Yoshie, H. (2005) Platelet-rich plasma combined with a porous hydroxyapatite graft for the treatment of intrabony periodontal defects in humans: A comparative controlled clinical study. Journal of Periodontology, 76, 890-898. doi:10.1902/jop.2005.76.6.890

[18] Kawase, T., Okuda, K., Kogami, H., Nakayama, H., Nagata, M., Sato, T., Wolff, L.F. and Yoshie, H. (2010) Human periosteum-derived cells combined with superporous hydroxyapatite blocks used as an osteogenic regenerative therapy: An animal implantation study using nude mice. Journal of Periodontology, 81, 420-427. doi:10.1902/jop.2009.090523

[19] Ender, A., Mörmann, W.H. and Mehl, A. (2010) Efficiency of a mathematical model in generating $\mathrm{CAD} / \mathrm{CAM}$ partial crowns with natural tooth morphology. Clinical Oral Investigations, 15, 283-289. doi:10.1007/s00784-010-0384-Z

[20] Yamamiya, K., Okuda K., Kawase, T., Hata K., Wolff, L. F. and Yoshie, H. (2008) Tissue-engineered cultured periosteum used with platelet-rich plasma and hydroxyapatite in treating human osseous defects. Journal of Periodontology, 79, 811-818. doi:10.1902/jop.2008.070518

[21] Okuda, K., Yamamiya, K., Kawase, T., Mizuno, H., Ueda, M. and Yoshie, H. (2009) Treatment of human infrabony periodontal defects by grafting human cultured periosteum sheets combined with platelet-rich plasma and porous hydroxyapatite granules: Case series. Journal of the Internatinoal Academy of Periodontology, 11, 206-213. 